\title{
Certain Expansions in the Algebra of Quantum Mechanics ${ }^{1}$
}

\author{
By Neal H. McCoy (Smith College, Northampton, Mass., U.S.A.) \\ Communicated by W. H. McCrea.
}

(Received 12th March 1932. Read 6th May 1932.)

§1. Introduction. The algebra of quantum mechanics ${ }^{2}$ is characterized by the fact that the variables $p, q$ obey all the laws of ordinary algebra except that multiplication is non-commutative and instead there exists a relation of the form

$$
p q-q p=c
$$

where $c$ is a real or complex scalar constant and is thus commutative with both $p$ and $q$.

It is well known that the relation (1) may be satisfied by certain infinite matrices, in which case $c$ is to be interpreted as a scalar matrix. It is also satisfied by the differential operators,

$$
p=c d / d x, \quad q=x,
$$

where $x$ is a real variable. We shall make use of this fact in $\S 5$.

One of the interesting and difficult problems in this algebra is that of expressing functions of $p$ and $q$ in some kind of a standard or normal form. We shall say, for convenience, that a function is in normal form if it is of the type, $\Sigma f_{i}(q) g_{i}(p)$, in which all factors involving $q$ occur to the left of those involving $p$. Of course the other order could have been chosen just as well; and the results for one case lead readily to corresponding results for the other.

The purpose of this note is to indicate how the use of differential equations may aid in obtaining the normal form of a function. This device is then used to find the normal form of certain exponential functions, in which case the results are particularly simple. Finally in $\S 7$ we find by this method an expansion of $[p+\phi(q)]^{n}$ in normal form, a result which was previously obtained by Kermack and McCrea in a different manner.

1 Some of the results presented here were obtained while the author was a National Research Fellow at Princeton University.

2 For references to this algebra see a previous paper, Tiansactions of the American Mathemutical Society, 31 (1929), 793-806 ; also Kermack and McCrea, Proc. Edinburgh Math. Soc. (2), 2 (1931), 220-239. Kermack and McCrea used the relation obtained from (1) by placing $c=-1$. 
Although most of these results are formal in character they seem to be of sufficient interest to warrant a brief account.

§2. Use of partial differential equations. If $\phi$ is a function of $p$ and $q$, we may define partial derivatives in the following manner ${ }^{1}$

and

$$
\begin{aligned}
& p \phi-\phi p=c \frac{\partial \phi}{\partial q} \\
& \phi q-q \phi=c \frac{\partial \phi}{\partial p} .
\end{aligned}
$$

It follows from these relations that with the proper care in preserving the order of factors, the usual formulae for differentiation hold. For example,

$$
\frac{\partial}{\partial p}\left(p^{2} q p^{3} q\right)=3 p^{2} q p^{2} q+2 p q p^{3} q .
$$

These results may be established for polynomials by induction, and are thus formally correct for infinite series. We assume their validity for the functions to be considered below.

If $f(p, q)$ is a given function of $p$ and $q$, let $f_{N}(p, q)$ denote the normal form of $f(p, q)$. For example, if $f(p, q)=(p+q)^{2}$, then

$$
f(p, q)=f_{N}(p, q)=p^{2}+2 q p+q^{2}+c,
$$

by relation (1).

Suppose it has been found in some way that a given function $f(p, q)$ satisfies one or more differential equations of the form

$$
\Sigma a_{i j}(q) \frac{\partial^{i+j} f}{\partial q^{i} \partial p^{j}}+\Sigma \frac{\partial^{i+j} f}{\partial q^{i} \partial p^{j}} \beta_{i j}(p)+\Sigma \gamma_{i j}(q) \frac{\partial^{i+j} f}{\partial q^{i} \partial p^{j}} \delta_{i j}(p)=0 .
$$

Then $f_{N}(p, q)$ will also satisfy (4) and in verifying this fact no interchange of order is necessary. Hence $f_{N}(y, x)$ satisfies

$$
\Sigma\left[a_{i,}(x)+\beta_{i j}(y)+\gamma_{i j}(x) \delta_{i j}(y)\right] \frac{\partial^{i+i} f}{\partial x^{i} \partial y^{i}}=0,
$$

where $x$ and $y$ are ordinary commutative variables. It is understood that $f_{N}(y, x)$ is obtained from $f_{N}(p, q)$ by replacing $q$ by $x$, and $p$ by $y$. Thus $f_{N}(y, x)$ is to be found among the solutions of the system (5), a fact which often proves to be of value in finding the normal form of a function. In particular, for the cases considered in the next two sections, this fact alone serves to determine $f_{N}(y, x)$ to within a

1 Born, Heisenberg and Jordan, ZS. f. Physik, 35 (1926), 563. 
constant factor, which is then determined by special methods. After obtaining $f_{N}(y, x)$ we may clearly find $f_{N}(p, q)$ by writing $f_{N}(y, x)$ in such a form that in each term the $x$-factors occur to the left of the $y$-factors, and then replacing $x$ by $q, y$ by $p$.

As an example which will be needed below, suppose that our given function $f(p, q)$ satisfies the equations

$$
\begin{aligned}
& \frac{\partial f}{\partial p}=X f p+Y q f, \\
& \frac{\partial f}{\partial q}=Y f p+Z q f,
\end{aligned}
$$

where $X, Y, Z$ are constants. These equations correspond to (4) above. In this case it is readily verified that any solution of the corresponding equations of type (5) is of the form

Hence

$$
F(y, x)=\text { const. } \times\left[\exp \left(Z x^{2} / 2+Y x y+X y^{2} / 2\right)\right] .
$$

$$
f_{N}(p, q)=K e^{Z_{q^{2}} / 2} e^{Y_{q_{1}} p} e^{X_{p^{2}} / 2},
$$

where $K$ is a properly chosen constant and

$$
e^{Y_{q_{1} p}}=1+Y q p+Y^{2} q^{2} p^{2} / 2 !+Y^{3} q^{3} p^{3} / 3 !+\ldots
$$

The significance of the notation $q_{1}$ is merely that the $q$-factors are to come first, that is on the left, in each term.

§3. The function $e^{p+q}$. Let us consider first the function

$$
e^{p+q}=1+(p+q)+(p+q)^{2} / 2 !+\ldots
$$

One could of course find the normal form of $(p+q)^{n}$, and from it get the normal form of the function (7). Indeed this is the method successfully used by Kermack and McCrea. ${ }^{1}$ However we shall now find the normal form of the exponential function ( 7 ) in a formal mannner without the use of the normal form of $(p+q)^{n}$.

It is easily seen by induction on $n$ that

$$
\frac{\partial}{\partial q}(p+q)^{n}=\frac{\partial}{\partial p}(p+q)^{n}=n(p+q)^{n-1} .
$$

Hence $e^{i+q}$ satisfies the partial differential equations

$$
\frac{\partial f}{\partial q}=f, \frac{\partial f}{\partial p}=f
$$

1 Loc. cit., 224. 
It follows from our previous discussion that

$$
e^{i+q}=\left(e^{p+1}\right)_{N}=K e^{q} e^{p},
$$

where $K$ is a properly chosen constant.

We may calculate $K$ directly in the following way. We have

$$
(p+q)^{n+1}=(p+q)(p+q)^{n}=q(p+q)^{n}+(p+q)^{n} p+c n(p+q)^{n-1},
$$

by (3). Thus the constant term in the normal form of $(p+q)^{n+1}$ is the constant term in the normal form of $(p+q)^{n-1}$ multiplied by $c n$. But the constant term in the normal form of $(p+q)$ is 0 , in $(p+q)^{2}$ is $c$. Hence

$$
\begin{aligned}
K & =1+c / 2+(c / 2)^{2} / 2 !+(c / 2)^{3} / 3 !+\ldots \\
& =e^{c / 2} .
\end{aligned}
$$

We have thus the final result

$$
e^{p+q}=e^{c / 2} e^{t} e^{p},
$$

which was obtained by Kermack and McCrea. ${ }^{1}$

The constant $K$ may also be obtained by using the special interpretation (2) of $p$ and $q$, and operating on a properly chosen function of $x$. Let the notation $A \rightarrow f(x)$ mean the result of operating on $f(x)$ with the operator $A$. Then

Hence

$$
(c d / d x+x) \rightarrow e^{\left(x-x^{2} / 2\right) / c}=e^{\left(x-x^{2} / 2\right) / c} .
$$

$e^{e^{d \frac{d}{d x}+x} \rightarrow e^{\left(x-x^{2} / 2\right) / c}}=e e^{\left(x-x^{2} / 2\right) / c}=K e^{x} e^{e \frac{d}{d \cdot c} \rightarrow e^{\left(x-x^{2} / 2\right) / c}}=K e^{x} e^{\left.\left[(x+c)-(x+c)^{2} / 2\right]\right] c}$, from which we find $K=e^{c / 2}$.

§4. The function $\exp \left[a p^{2}+b q p+d q^{2}\right]$. We pass now to the determination of the normal form of the exponential function of a general quadratic function of $p$ and $q$, which is of considerably more difficulty than that of the linear function.

We first remark that if $a, b, d$ are real or complex constants, then we have

$$
a p^{2}+b q p+d q^{2}=(a p+\beta q)(\gamma p+\delta q)+\eta c,
$$

where the constants $a, \beta, \gamma, \delta, \eta$ are properly determined This is clearly the case if $a=0$. If $a \neq 0$, then the relation is satisfied if we place

$$
a=1, \beta=\left[b-\left(b^{2}-4 a d\right)^{\frac{1}{2}}\right] /(2 a), \gamma=a, \delta=-\eta=\left[b+\left(b^{2}-4 a d\right)^{\frac{1}{2}}\right] / 2 .
$$

1 This result was obtained independently by the present author by this method prior to the publication of their paper. 
We may thus, without loss of generality, confine our attention to the function, $\exp [(\alpha p+\beta q)(\gamma p+\delta q)]$

$$
=\mathbf{1}+[(\alpha p+\beta q)(\gamma p+\delta q)]+[(\alpha p+\beta q)(\gamma p+\delta q)]^{2} / 2 !+\ldots .
$$

where $a, \beta, \gamma, \delta$ are real or complex numbers. We now assume that $a, \beta, \gamma, \delta$ are arbitrary parameters. After we have found the normal form of the function (10) for this case, we may find the corresponding expression in the case of special values of these constants by a limiting process.

Let us denote the function (10) by $f$. We seek first of all the partial derivatives of $f$, and are led to a set of differential equations satisfied by this function. We have immediately that

$[(\alpha p+\beta q)(\gamma p+\delta q)]^{n}(\alpha p+\beta q)=(\alpha p+\beta q)[(\gamma p+\delta q)(\alpha p+\beta q)]^{n}$, and thus

$$
f(a p+\beta q)=(a p+\beta q) \exp [(\gamma p+\delta q)(\alpha p+\beta q)] .
$$

But it is easily found that

$$
(\gamma p+\delta q)(\alpha p+\beta q)=(\alpha p+\beta q)(\gamma p+\delta q)-c(\alpha \delta-\beta \gamma) .
$$

Let us write $D \equiv(\alpha \delta-\beta \gamma)$. Then

$$
\exp [(\gamma p+\delta q)(\alpha p+\beta q)]=e^{-c D} f
$$

and substituting in (12) we get

$$
\alpha(f p-p f)+\beta(f q-q f)=\left(e^{-c D}-1\right)(\alpha p+\beta q) f .
$$

By relations (3) this may be written in the form

or

$$
-c \alpha \frac{\partial f}{\partial q}+c \beta \frac{\partial f}{\partial p}=\left(e^{-c D}-1\right)\left[\alpha\left(f p+c \frac{\partial f}{\partial q}\right)+\beta q f\right],
$$

$$
\beta \frac{\partial f}{\partial p}-\alpha e^{-c D} \frac{\partial f}{\partial q}=(1 / c)\left(e^{-c D}-1\right)(\alpha f p+\beta q f) .
$$

If in relation (11) we interchange $(\alpha p+\beta q)$ and $(\gamma p+\delta q)$ and proceed as above, we find that $f$ also satisfies the equation,

$$
\gamma \frac{\partial f}{\partial q}-\delta e^{-c D} \frac{\partial f}{\partial p}=(1 / c)\left(e^{-c D}-1\right)(\gamma f p+\delta q f) .
$$

We may now solve (13) and (14) for $\frac{\partial f}{\partial p}$ and $\frac{\partial f}{\partial q}$, getting

$$
\begin{aligned}
& \frac{\partial f}{\partial p}=X f p+Y q f, \\
& \frac{\partial f}{\partial q}=Y f p+Z q f,
\end{aligned}
$$


where

$$
X=\frac{a \gamma}{c}\left[\frac{e^{-2 c D}-1}{\beta \gamma-a \delta e^{-2 c D}}\right], Y=-\frac{1}{c}\left[1+\frac{D e^{-c D}}{\beta \gamma-a \delta e^{-2 c D}}\right], \quad Z=\frac{\delta \beta}{a \gamma} X .
$$

It follows from $\S 2$ that

$$
f=K(c) e^{Z_{L^{2} / 2}} e^{\boldsymbol{Y}_{q_{1} t^{3}}} e^{\boldsymbol{X}_{p^{2} / 2}},
$$

where $K(c)$ is a constant to be determined.

§5. Calculation of $K(c)$. If

$$
[(\alpha p+\beta q)(\gamma p+\delta q)]^{n}
$$

is expressed in normal form by repeated use of relation (1), the constant term is of the form $a_{n} c^{n}$, where $a_{n}$ does not involve $c$ but is a function of $a, \beta, \gamma, \delta$. This may be verified by the following device which often proves useful. Let us assign to both $p$ and $q$ the weight 1 so that, by relation (1), $c$ is of weight 2 . Our function (17) is clearly isobaric of weight $2 n$, and each term in the normal form must also be of this weight as the use of relation (1) makes no change in the weights. Hence the constant term must be of the required form .

Let us now introduce the special interpretation, $q=x, p=c d / d x$, and denote by $A$ the expression

$$
\left(a c \frac{d}{d x}+\beta x\right)\left(\gamma c \frac{d}{d x}+\delta x\right) \text {. }
$$

Then

$$
A \rightarrow 1=\delta\left(c \alpha+\beta x^{2}\right) \text {, }
$$

and thus

$$
A^{n} \rightarrow 1=A^{n-} \rightarrow \delta\left(c \alpha+\beta x^{2}\right) .
$$

The term in $A^{n} \rightarrow 1$ not containing $x$ is exactly the constant in the normal form of the function (17) and thus is of the form $a_{n} c^{n}$. Let $P_{1}(c)$ denote the constant in $e^{f} \rightarrow 1$, that is

$$
P_{1}(c)=\sum_{n=0}^{\infty} a_{n} c^{n} / n !
$$

Similarly let $P_{2}(c)$ be the constant in $e^{A} \rightarrow \delta\left(c \alpha+\beta x^{2}\right)$. Then

Thus we have

$$
P_{2}(c)=\sum_{n=0}^{\infty} a_{n+1} c^{n+1 / n !} \text {. }
$$

$$
P_{2}(c)=c P_{1}^{\prime}(c),
$$

where the dash denotes differentiation with respect to $c$. But from relation (16) we find $P_{1}(c)=K(c)$. Also

$$
e^{A} \rightarrow \delta\left(c \alpha+\beta x^{2}\right)=K(c) \delta\left(c \alpha+c^{2} \beta x\right)+\text { terms involving } x .
$$


Hence $P_{2}(c)=K(c) \delta\left(c \alpha+c^{2} \beta X\right)$, and we get the result,

$$
K^{\prime}(c)=K(c) \delta(\alpha+c \beta X) .
$$

Hence if we write $X(c)$ for $X$, we find that

$$
K(c)=\exp \left[\delta \alpha c+\beta \delta \int_{0}^{c} t X(t) d t\right] .
$$

The constant of integration has been determined by the fact that $\lim _{c \rightarrow 0} K(c)=1$. This is clear since when $c=0, p$ and $q$ are commutative.

We proceed to calculate the integral occurring in (19), that is

$$
\begin{aligned}
\alpha \gamma \int_{0}^{c} \frac{\left(e^{-2 t D}-1\right) d t}{\beta \gamma-\alpha \delta e^{-2 t D}} & =-\alpha \gamma \int_{0}^{c}\left[\frac{1}{\alpha \delta}+\frac{D}{\alpha \delta\left(\beta \gamma-\alpha \delta e^{-2 t D}\right)}\right] d t \\
& =-\frac{\gamma}{\delta} c-\frac{\gamma D}{\delta} \int_{0}^{c} \frac{d t}{\beta \gamma-\alpha \delta e^{-2 t D}} .
\end{aligned}
$$

By making the substitution, $z=\beta \gamma-\alpha \delta e^{-2 t D}$, we may find the value of the integral occurring in this expression to be

$$
\frac{1}{2 D \beta_{\gamma}} \log \left(\frac{\alpha \delta e^{-2 c D}-\beta \gamma}{D}\right)+\frac{c}{\beta \gamma} .
$$

Substituting back in (20) and (19) we get,

$$
K(c)=\left[\frac{D}{a \delta e^{-2 c D}-\beta \gamma}\right]^{1 / 2}
$$

the determination of the square root being such that $\lim _{c \rightarrow 0} K(c)=1$. We collect these results as follows :

Theorem. If $p q-q p=c$, and $\alpha, \beta, \gamma, \delta$ are real or complex constants, then

$$
\exp [(\alpha p+\beta q)(\gamma p+\delta q)]=\left[\frac{D}{\alpha \delta e^{-2 c D}-\beta \gamma}\right]^{1 / 2} e^{Z_{1} / 2} e^{\boldsymbol{Y}_{q_{1} p^{\prime}}} e^{X_{p^{4} / 2}}
$$

where

and

$$
\begin{aligned}
& D=(\alpha \delta-\beta \gamma), X=\frac{\alpha \gamma}{c}\left[\frac{e^{-2 c D}-1}{\beta \gamma-\alpha \delta e^{-2 c D}}\right], \\
& Y=-\frac{1}{c}\left[1+\frac{D e^{-c D}}{\beta \gamma-\alpha \delta e^{-2 c D}}\right], \quad Z=\frac{\delta \beta}{\alpha \gamma} X,
\end{aligned}
$$

$$
e^{Y_{q_{1} p}}=1+Y q p+Y^{2} q^{2} p^{2} / 2 !+Y^{3} q^{3} p^{3} / 3 !+\ldots
$$


\$6. Some special cases. Although we established this theorem for general $a, \beta, \gamma, \delta$, we may find the corresponding result for special values of these coefficients by a limiting process.

For example, consider $e^{(x+q)^{2}}$. Let us set $a=\beta=\gamma=1$, and let $\delta$ approach 1 as a limiting value. We find in this way that

$e^{(2+1)^{\prime \prime}}=(1-2 c)^{-\frac{1}{2}} \exp \left[q^{2} /(1-2 c)\right] \exp \left[2 q_{1} p /(1-2 c)\right] \exp \left[p^{2} /(1-2 c)\right]$

Other special cases of interest which may be obtained from our theorem are:

$$
e^{i^{\prime \prime}+i^{2}}=e^{i n} e^{\left.\left(p+i_{i}\right) p-i_{i}\right)}=
$$

$(\sec 2 c)^{\frac{1}{2}} \exp \left[q^{2} \tan 2 c /(2 c)\right] \exp \left[q_{1} p(\sec 2 c-1) / c\right] \exp \left[p^{2} \tan 2 c /(2 c)\right]$,

$$
\begin{aligned}
e^{a_{11^{\prime}}} & =\exp \left[\left(e^{r a t}-1\right) q_{1} p / c\right], \\
e^{a x^{\prime}} & =e^{c a} \exp \left[\left(e^{c a}-1\right) q_{1} p / c\right] .
\end{aligned}
$$

Each of these special cases may of course be obtained directly by the method of $\S 4$ without use of any limiting process. In particular, relation (24) may also be readily verified from a knowledge of the normal form of $(q p)^{n}$ which has been given by Schwatt ${ }^{1}$ for the special interpretation (2) of $p$ and $q$.

$\S 7$. The function $[p+\phi(q)]^{n}$. We conclude with the determination of the normal form of the function,

$$
g_{n}=[p+\phi(q)]^{n},
$$

$\phi(q)$ being a given polynomial in $q$, and $n$ a positive integer. A formula equivalent to that which we seek has been previously obtained by Kermack and McCrea in a different manner. ${ }^{2}$

We first seek the derivatives of $g_{n}$. It is easily verified by induction that

$$
\frac{c g_{n}}{c p}=n[p+\phi(q)]^{n-1}=n g_{n-1} .
$$

By considering the cases $n=1,2,3$, the following formula for $\frac{\partial g_{n}}{\partial q}$ suggests itself,

$$
\frac{\partial g_{n}}{\hat{\partial} q}=\sum_{s=1}^{n} c^{s-1}\left(\begin{array}{c}
n \\
s
\end{array}\right) \frac{\hat{c}^{s} \phi}{\hat{c} q^{s}} g_{n-8}
$$

1 I. J. Schwatt, Operations with Series (Univ. of Pennsylvania Press) (1924), chap. V.

¿ Loc. cit., 227 . 
We shall prove this formula by induction on $n$. We assume it for $g_{n}$ and show that it holds for $g_{n+1}$. Let us write $\phi$ for $\phi(q)$. Since $g_{n+1}=(p+\phi) g_{n}$, we have

$$
\begin{aligned}
\frac{\partial g_{n+1}}{\partial q} & =(p+\phi) \frac{\partial g_{n}}{\partial q}+\frac{\partial \phi}{\partial q} g_{n} \\
& =\sum_{s=1}^{n} c^{s-1}\left(\begin{array}{c}
n \\
s
\end{array}\right) \frac{\partial^{s} \phi}{\partial q^{s}} g_{n-s+1}+\sum_{s=1}^{n} c^{s}\left(\begin{array}{c}
n \\
s
\end{array}\right) \frac{\partial^{s+1} \phi}{\partial q^{s+1}} g_{n-s}+\frac{\partial \phi}{\partial q} g_{n},
\end{aligned}
$$

by relations (28) and (3). From this we find that

$$
\begin{aligned}
\frac{\partial \boldsymbol{g}_{n+1}}{\partial q} & =\sum_{s=1}^{n} c^{s-1}\left[\left(\begin{array}{c}
n \\
s
\end{array}\right)+\left(\begin{array}{c}
n \\
s-1
\end{array}\right)\right] \frac{\partial^{s} \phi}{\partial q^{s}} g_{n-s+1}+c^{n} \frac{\partial^{n+1} \phi}{\partial q^{n+1}} \\
& =\sum_{s=1}^{n+1} c^{s-1}\left(\begin{array}{c}
n+1 \\
s
\end{array}\right) \frac{\partial^{s} \phi}{\hat{c} q^{s}} g_{n+1-s},
\end{aligned}
$$

which establishes the formula.

We now introduce the symbol $Q \equiv \frac{\partial}{\partial p}$, with the understanding that it is to operate on every function on the right of it in any given term in which it appears. We may then write the equation (28) in the form

$$
\frac{\partial g_{n}}{\partial q}=\sum_{s=1}^{n} \frac{c^{s-1}}{s !} \frac{\partial^{s} \phi}{\partial q^{s}} Q^{s} g_{n}
$$

or, symbolically, $g_{n}$ satisfies the equation,

$$
\frac{\partial g}{\partial q}=\frac{1}{c}\left[\phi_{1}(q+c Q)-\phi_{1}(q)\right] g,
$$

where the subscript is placed on the $\phi$ merely to emphasize that all $\phi$-factors are to be on the left in each term.

By the discussion in $\S 2$, the normal form of the general solution of (29) is, in operational form,

$$
g=\left\{\exp \left[(1 / c) \int_{01}^{c Q} \phi_{1}(q+t) d t\right]\right\} \psi(p)
$$

$\psi(p)$ being an arbitrary function of $p$. This suggests that

$$
g_{n}=\left\{\exp \left[(1 / c) \int_{0}^{c Q} \phi_{1}(q+t) d t\right]\right\} \psi_{n}(p)
$$

if $\psi_{n}(p)$ is a properly chosen function of $p$. 
It is readily found that $g_{1}$ is of the form (30) with $\psi_{1}(p)=p$. Suppose now $g_{n}$ is of this form with $\psi_{n}(p)$ determined. Let $\bar{\phi}(t)$ denote. the indefinite integral of $\phi(t)$. Then we may write

$$
g_{n}=\left\{\exp \left[(1 / c) \bar{\phi}_{1}(q+c Q)\right] \cdot \exp \left[-(1 / c) \bar{\phi}_{1}(q)\right]\right\} \psi_{n}(p) .
$$

It may now be verified that $p$ is commutative with $(q+c Q)$ from the fact that $Q p-p Q=1$, and relation (1). We have also

$$
p e^{-(1 / c) \bar{\phi}(q)}=e^{-(1 / c) \bar{\phi}(q)} p-\phi(q) e^{-(1 / c) \bar{\phi}(q)}
$$

by relations (3). It is now readily verified that

$$
g_{n+1}=(p+\phi) g_{n}=\left\{\exp \left[(1 / c) \int_{0}^{c Q} \phi_{1}(q+t) d t\right]\right\} p \psi_{n}(p) .
$$

Hence $g_{n+1}$ is of the desired form if we choose $\psi_{n+1}(p)=p \psi_{n}(p)$. Thus $\psi_{n}(p)=p^{n}$ and we have the desired result,

$$
\begin{aligned}
{[p+\phi(q)]^{n} } & =\left\{\exp \left[(1 / c) \int_{0}^{c Q} \phi_{1}(q+t) d t\right]\right\} p^{n} \\
& =\left\{\exp \left[\phi_{1}(q) \frac{\partial}{\partial p}+\frac{c}{2 !} \frac{\partial \phi_{1}(q)}{\partial q} \frac{\partial^{2}}{\partial p^{2}}+\frac{c^{2}}{3 !} \frac{\partial^{2} \phi_{1}(q)}{\partial q^{2}} \frac{\partial^{3}}{\partial p^{3}}+\ldots\right]\right\} p^{n} .
\end{aligned}
$$

